\title{
Sur la représentation conforme des surfaces de Riemann à bord et une caractérisation des courbes séparantes
}

\author{
Alexandre Gabard
}

Résumé. On présente une démonstration simplifiée d'un théorème d'Ahlfors : étant donné une courbe algébrique réelle séparante, il est toujours possible d'exhiber un morphisme vers la droite dont les fibres au-dessus des points réels sont toutes exclusivement formées de points réels. Ensuite, on montre comment notre méthode, basée sur l'usage du théorème d'Abel, conduit à une meilleure borne sur le degré d'un tel morphisme que celle obtenue par Ahlfors.

\begin{abstract}
We present a simplified proof of a theorem due to Ahlfors : given a dividing real algebraic curve, it is always possible to find a morphism to the line such that the fibers over real points consist only of real points. Then in a second part, we show how our method - which is quite elementary, relying only on the use of Abel's theorem - even leads to a better bound on the degree of such a morphism than the one obtained by Ahlfors.
\end{abstract}

Codes AMS (2000). 14P25, 30F50, 14H40, 14C20.

Mots clés. Courbes algébriques réelles séparantes, morphismes vers la droite, Théorème d'Abel, jacobienne, surfaces de Riemann à bord.

\section{Introduction}

Soit $C$ une courbe algébrique réelle projective, lisse et géométriquement irréductible de genre $g$. La conjugaison complexe induit une involution $\sigma$ sur le lieu complexe $C(\mathbb{C})$, qui fixe le lieu réel $C(\mathbb{R})$. Il en résulte que le lieu imaginaire $C(\mathbb{C})-C(\mathbb{R})$ est soit connexe, ou formé de deux composantes, auquel cas la courbe $C$ est dite séparante. Une courbe réelle est donc soumise à la dichotomie (dévoilée par Klein) être ou ne pas être séparante (cf. [K], p. 565). Une question qui apparait alors comme centrale est de disposer de critères pour la reconnaissance du caractère séparant ou non d'une courbe réelle donnée. Le point de départ du présent exposé est que si une courbe admet un morphisme vers la droite $\mathbb{P}^{1}$ (qui est l'exemple le plus primitif de courbe séparante) satisfaisant à une condition bien particulière, alors le caractère séparant de la droite se transmet à la courbe, d'où un critère de séparation. L'objet de cette note est de montrer que ce critère est infaillible, en ce sens qu'il permet de 
déceler toutes les courbes séparantes. De façon précise, nous allons montrer : dire qu'une courbe réelle est séparante équivaut à l'existence d'un morphisme vers $\mathbb{P}^{1}$ qui prend des valeurs réelles seulement sur le lieu réel de la courbe. Ainsi la droite apparaît comme l'archétype de toute courbe séparante, et il est remarquable de noter qu'une condition purement topologique s'avère être rigoureusement équivalente à une condition algébro-géométrique.

Note bibliographique. En fait, comme je l'ai appris grâce aux articles de AllingGreenleaf $[\mathrm{AG}]$ et de Geyer-Martens [GM], un résultat équivalent fut démontré par L.V. Ahlfors en 1950, qui déduit d'un problème d'extrémalisation la possibilité de représenter toute surface de Riemann à bord ${ }^{1}$ compacte comme revêtement holomorphe (ramifié) du disque (cf. [Ah], §4, pp. 122-133). [L'équivalence de cet énoncé avec le nôtre résulte de la correspondance naturelle (induite par double de Schottky) entre surfaces de Riemann à bord et surfaces de Riemann munies d'une involution antiholomorphe dont le lieu fixe disconnecte la surface.] Ce résultat d'Ahlfors est en fait l'aboutissement d'une longue série de travaux en théorie de la représentation conforme, dont l'origine est le Riemann mapping theorem, et dont les généralisations successives sont dues à Riemann lui-même pour les domaines circulaires [Rie], puis Schottky [Sch], Bieberbach [Bi] et Grunsky [Gr1], [Gr2] pour les domaines multiplement connexes quelconques.

On propose ici un argument on l'espère plus direct qui, moyennant le seul usage du théorème d'Abel, ramène cette question d'existence au simple fait topologique ; selon lequel tout point d'un tore $T^{g}=(\mathbb{R} / \mathbb{Z})^{g}$ peut s'écrire comme somme de $g$ points situés sur $g$ courbes fondamentales, i.e. qui forment une base de l'homologie de $T^{g}$. Notre approche illustre donc une fois de plus la puissance des méthodes topologiques pour établir des résultats d'existence en géométrie algébrique.

Dans un premier temps on retrouve exactement le même degré $g+1$ que celui obtenu par Ahlfors. Ensuite en raffinant l'étude, on obtient une amélioration quantitative qui reflète parfaitement la topologie de la surface de Riemann à bord, qui est déterminée par les deux entiers $p$ (= le genre de la surface à bord) et $r(=$ le nombre de composantes du bord), en montrant qu'il est possible d'abaisser le degré $r+2 p$ obtenu par Ahlfors à $r+p$. Cette amélioration confirme une conjecture de Nehari et Tietz (cf. [Neh] et [Tie], p. 49). Notre travail laisse cependant ouverte la question de savoir si l'amélioration ainsi obtenue est optimale.

\section{L'exemple de la Gürtelkurve}

On commence par décrire l'exemple qui a motivé originellement ce travail. On considère une quartique $C_{4} \subset \mathbb{P}^{2}$ réelle obtenue en lissifiant une paire de coniques réelles

\footnotetext{
${ }^{1}$ C'est-à-dire un espace topologique séparé holomorphiquement modelé sur le demi-plan fermé (pour une définition précise cf. [AS], p. 117).
} 
comme l'indique la Figure 1. On se demande si cette courbe, que Klein appelle la Gürtelkurve, est ou non séparante.

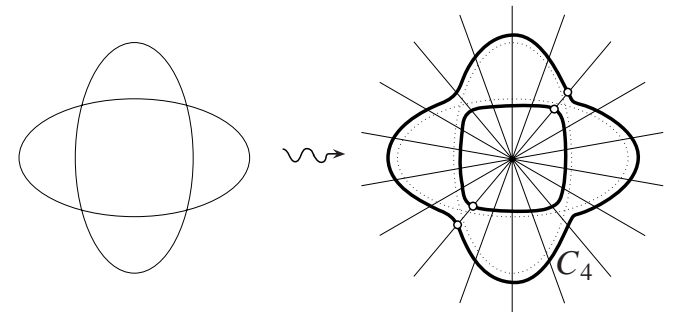

Figure 1

Proposition 2.1. La Gürtelkurve est séparante.

Preuve. Les membres réels du pinceau des droites passant par un point réel choisi à l'intérieur de l'ovale le plus profond du nid (cf. Figure 1), découpent sur la courbe $C_{4}$ seulement des points réels. Le morphisme correspondant $f: C_{4} \rightarrow \mathbb{P}^{1}$ possède donc des fibres au-dessus des points réels qui sont toutes seulement formées de points réels. Par suite $f$ induit une flèche continue $C_{4}(\mathbb{C})-C_{4}(\mathbb{R}) \rightarrow \mathbb{P}^{1}(\mathbb{C})-\mathbb{P}^{1}(\mathbb{R})$ entre

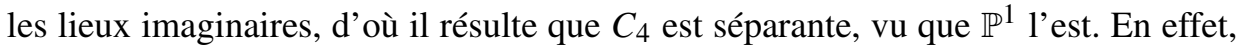
sinon $C_{4}(\mathbb{C})-C_{4}(\mathbb{R})$ serait connexe, mais alors l'image continue d'un connexe étant connexe, son image serait entièrement contenue dans un hémisphère de la sphère $\mathbb{P}^{1}(\mathbb{C})$. Mais ceci est incompatible avec le fait que cette image doit être stable sous la conjugaison complexe, puisque le morphisme $f$ étant défini sur $\mathbb{R}$, il induit une application équivariante pour les conjugaisons complexes.

\section{Morphismes saturés et formulation du problème d'existence}

Définition 3.1. Un morphisme $f: C \rightarrow \mathbb{P}^{1}$ défini sur $\mathbb{R}$ est dit saturé si ses fibres au-dessus des points réels sont toutes seulement formées de points réels.

Remarque 3.2. Observons qu'un tel morphisme ne peut avoir de point de ramification réel. [En effet le comportement local de $f$ est donné par l'application de $\mathbb{C}$ dans $\mathbb{C}$, $z \mapsto z^{k}$ avec $k$ entier, dont les fibres au-dessus des points réels sont toutes réelles seulement lorsque $k=1$.] Par suite $f(\mathbb{R}): C(\mathbb{R}) \rightarrow \mathbb{P}^{1}(\mathbb{R})$ est un revêtement topologique du cercle. En particulier le degré $d$ d'un morphisme saturé $f: C \rightarrow \mathbb{P}^{1}$ est toujours au moins égal au nombre $r$ de composantes de $C(\mathbb{R})$.

Le raisonnement ci-dessus pour la Gürtelkurve fournit plus généralement le critère suivant : 
Proposition 3.3. Si une courbe réelle $C$ admet un morphisme saturé vers $\mathbb{P}^{1}$, alors $C$ est séparante ${ }^{2}$.

On est alors tenté par la réciproque :

Problème 3.4. Pour $C$ une courbe séparante, existe-t-il toujours un morphisme saturé de $C$ vers $\mathbb{P}^{1}$ ?

Dans ce qui suit, on va montrer que la réponse à cette question est affirmative.

\section{Le cas facile des courbes Harnack-maximales}

On propose d'abord de résoudre le Problème 3.4 pour les courbes qui réalisent la borne de Harnack, i.e. celles avec $r=g+1$ ovales (i.e. une composante de la partie réelle). Ces courbes sont automatiquement séparantes, car le genre peut se définir comme le nombre maximal de courbes disjointes que l'on peut ôter (récursivement) à la surface sans la morceller.

Proposition 4.1. Soit $C$ une courbe réelle avec $r=g+1$ (et donc séparante). $\mathrm{Il}$ existe alors un morphisme saturé $f: C \rightarrow \mathbb{P}^{1}$ de degré $g+1$.

Preuve. On rappelle que tout groupe (i.e. un diviseur effectif) $D_{0}$ de $d=g+1$ points sur une courbe $C$ de genre $g$ bouge, i.e. $\operatorname{dim}\left|D_{0}\right| \geq 1$ [ceci résulte de l'inégalité de Riemann $\left.\operatorname{dim}\left|D_{0}\right| \geq \operatorname{deg}\left(D_{0}\right)-g\right]$. Vu que $r=g+1$, on peut choisir $D_{0}$ un groupe de $d=g+1$ points avec un point sur chaque composante de $C(\mathbb{R})$, cf. Figure 2. On choisit ensuite une série $g_{d}^{1}$ réelle dans $\left|D_{0}\right|$ contenant $D_{0}$. Quitte à

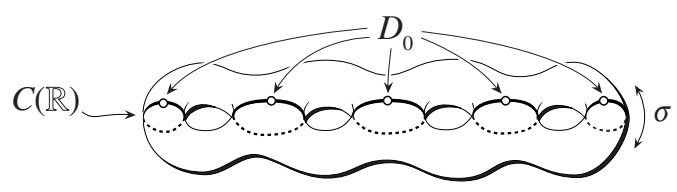

Figure 2

lui soustraire son éventuel lieu base $B$, on obtient une $g_{\delta}^{1}$ qui induit un morphisme $f: C \rightarrow \mathbb{P}^{1}$ réel. Lorsqu' on bouge $D_{0}-B$ dans le cercle $g_{\delta}^{1}(\mathbb{R})$, on génère une famille de diviseurs symétriques dont les points restent par continuité prisonniers de leurs ovales respectifs. Ceci montre déjà que $f$ est saturé de degré $\delta \leq g+1$. Or d'après la Remarque 3.2, le degré de $f$ est au moins égal au nombre d'ovales $r=g+1$, et lui est donc égal.

\footnotetext{
${ }^{2}$ On peut bien sûr dans cet énoncé remplacer $\mathbb{P}^{1}$ par une courbe séparante quelconque.
} 
Note. En fait ce raisonnement par continuité apparaît déjà dans [EC], Libro Terzo p. 264; mais ces derniers ne s'intéressant pas à la notion de morphisme saturé ils se contentent d'en tirer une démonstration de la borne de Harnack. Par contre l'énoncé ci-dessus figure explicitement dans un travail de J. Huisman (cf. [Hui], Prop. 3.2, p. 87), ainsi que dans un article de Bieberbach (cf. [Bi]).

Remarque. Malheureusement cet argument ne s'étend pas aux courbes non Harnackmaximales, car alors en choisissant nos $g+1$ points sur les $r$ ovales, le principe des tiroirs nous contraint d'en disposer deux au moins sur un même ovale; d'où une "surpopulation" qui nous expose à une éventuelle collision entre deux tels points, qui après s'être produite les laisse s'échapper dans le lieu imaginaire, ruinant notre tentative "bon marché" de construction d'un morphisme saturé.

\section{Reformulation du problème au moyen de l'équivalence linéaire}

Ce paragraphe est une simple traduction de la condition d'existence d'un morphisme saturé en termes de l'équivalence linéaire sur les groupes de points de la courbe. Réunissons d'abord quelques :

Définitions 5.1. Si $C$ est séparante, une moitié de $C$ est l'une des deux composantes de $C(\mathbb{C})-C(\mathbb{R})$. Un diviseur effectif est dit unilatéral si son support est entièrement contenu dans une moitié de $C$.

Pour résoudre le Problème 3.4, on va exploiter l'observation suivante :

Lemme 5.2. Soit $C$ une courbe séparante. L'existence d'un morphisme saturé $f: C \rightarrow \mathbb{P}^{1}$ équivaut à celle d'un diviseur unilatéral $D$ sur $C$ linéairement équivalent à son conjugué $D^{\sigma}$.

Preuve. $[\Rightarrow]$ Si $f: C \rightarrow \mathbb{P}^{1}$ est un morphisme saturé, $f$ induit une application $C(\mathbb{C})-C(\mathbb{R}) \rightarrow \mathbb{P}^{1}(\mathbb{C})-\mathbb{P}^{1}(\mathbb{R})$ continue entre les lieux imaginaires, et applique donc chaque moitié de $C$ sur une moitié de $\mathbb{P}^{1}$. Le pull-back $f^{*}(q)$ d'un point imaginaire $q \in \mathbb{P}^{1}(\mathbb{C})-\mathbb{P}^{1}(\mathbb{R})$ est donc un diviseur unilatéral $D$ tel que $D \sim D^{\sigma}$.

$[\Leftarrow]$ Réciproquement si un tel diviseur $D$ existe, on peut parler de la $g_{d}^{1}$ engendrée par $D$ et $D^{\sigma}$, qui est réelle et sans point base (vu que les supports de $D$ et $D^{\sigma}$ sont disjoints). Le morphisme correspondant $f: C \rightarrow g_{d}^{1}$ est donc réel. En particulier $g_{d}^{1} \cong \mathbb{P}^{1}$ est la droite réelle standard, vu que $C(\mathbb{R}) \neq \varnothing$, puisque $C$ est supposée séparante. Il en résulte que lorsque $D$ circule dans $g_{d}^{1}$ en évitant $g_{d}^{1}(\mathbb{R})$, on génère des diviseurs dont les points n'ayant le droit de franchir $C(\mathbb{R})$, restent dans la moitié de $C$, qui contient $D$. Ainsi en laissant dégénérer $D$ sur un point $D_{0} \in g_{d}^{1}(\mathbb{R})$ situé sur l'équateur (cf. Figure 3), on voit que $D_{0}$ est limite de diviseurs unilatéraux ; mais 


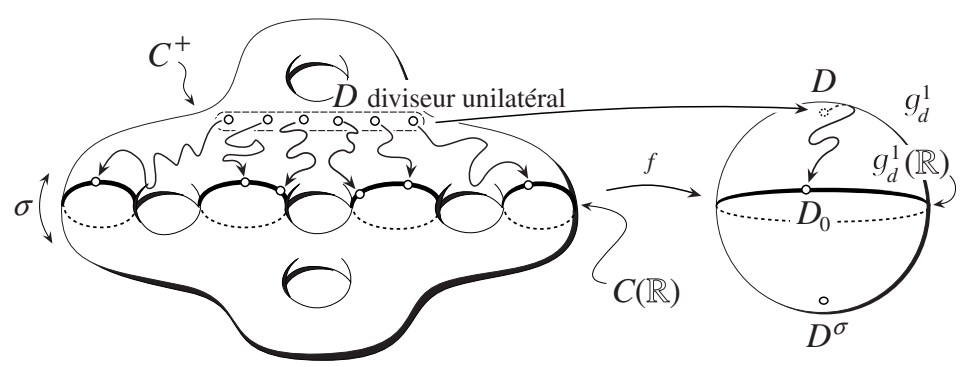

Figure 3

étant aussi symétrique, il en résulte que $D_{0}$ est seulement formé de points réels. Enfin $D_{0}$ pouvant être arbitrairement choisi dans $g_{d}^{1}(\mathbb{R})$, il s'ensuit que le morphisme $f$ est saturé.

Tout revient donc à exhiber un diviseur unilatéral $D$ tel que $D \sim D^{\sigma}$. Pour ce faire on va s'appuyer sur le théorème d'Abel, qui permet de traîter les questions d'équivalence linéaire en termes du plongement de la courbe dans sa jacobienne : deux diviseurs de même degré sont linéairement équivalents si et seulement si les sommes de leurs points vus dans la jacobienne coüncident.

\section{Résolution du problème via le théorème d'Abel}

Notons $J=\mathbb{C}^{g} / \Lambda$ la jacobienne de la courbe donnée $C$, où $\Lambda$ est le réseau des périodes d'une base fixée $\omega_{1}, \ldots, \omega_{g}$ de l'espace des 1-formes holomorphes sur $C$, i.e. $\Lambda=\left\{\left(\int_{\gamma} \omega_{1}, \ldots, \int_{\gamma} \omega_{g}\right) \in \mathbb{C}^{g} \mid \gamma \in H_{1}(C(\mathbb{C}), \mathbb{Z})\right\}$. Le morphisme d'Abel $\alpha: C \rightarrow J$ est défini par $\alpha(p)=\left(\int_{p_{0}}^{p} \omega_{1}, \ldots, \int_{p_{0}}^{p} \omega_{g}\right)$ où $p_{0} \in C(\mathbb{C})$ est un point base.

Vu qu'on suppose $C$ définie sur $\mathbb{R}$, on peut choisir une base $\omega_{1}, \ldots, \omega_{g}$ formée de différentielles réelles. [Dire qu'une différentielle $\omega$ est réelle signifie que la fonctionnelle d'intégration correspondante $\int \omega$ est équivariante pour la conjugaison complexe, i.e. $\int_{\gamma^{\sigma}} \omega=\overline{\int_{\gamma} \omega}$.] La jacobienne $J$ hérite alors d'une structure réelle, induite par le produit de $g$ conjugaisons complexes $\sigma: \mathbb{C}^{g} \rightarrow \mathbb{C}^{g}$, vu que l'hypothèse de réalité faite sur les $\omega_{i}$ entraîne la stabilité du réseau $\Lambda$ par $\sigma$. Si en outre on choisit $p_{0}$ réel (ce qui est loisible, puisqu'on suppose $C$ séparante), le morphisme $\alpha$ est défini sur $\mathbb{R}$, c'est-à-dire équivariant pour les conjugaisons complexes.

Cela étant, la condition requise $D \sim D^{\sigma}$ équivaut d'après le théorème d'Abel à $\alpha(D)=\alpha\left(D^{\sigma}\right)$ [où $\alpha(D)$ est défini par additivité], ou encore $\alpha$ étant équivariant à $\alpha(D)=\alpha(D)^{\sigma}$, i.e. à ce que $\alpha(D) \in J(\mathbb{R})$ soit un point réel de la jacobienne. Notre 
problème revient à trouver $d$ points $P_{1}, \ldots, P_{d} \in J$ situés dans $\alpha\left(C^{+}\right)$[où $C^{+}$est une moitié de notre courbe séparante] et tels que leur somme $P_{1}+\cdots+P_{d} \in J(\mathbb{R})$. Au niveau du groupe quotient $T^{g}=J / J(\mathbb{R})$, il s'agit alors d'exprimer 0 comme somme de d points $\pi_{1}, \ldots, \pi_{d}$ appartenant au sous-ensemble $\Omega=\pi\left(\alpha\left(C^{+}\right)\right)$de $T^{g}$, où $\pi: J \rightarrow T^{g}$ est la projection canonique. Le quotient $T^{g}$ est un groupe de Lie réel connexe compact abélien de dimension $g$, et est donc isomorphe au tore $(\mathbb{R} / \mathbb{Z})^{g}$.

Il reste donc à préciser le plongement de $\Omega$ dans $T^{g}$; nous allons le faire en montrant que $\Omega \cup\{0\}$ contient une base de l'homologie en dimension 1 du tore $T^{g}$. Ce renseignement bien que partiel sera suffisant pour notre objet.

Preuve de ce renseignement. On commence par choisir une base de $H_{1}(C(\mathbb{C}), \mathbb{Z})$ adaptée à l'involution $\sigma$ comme l'indique la Figure 4; constituée des 1-cycles suivants :

$$
\begin{gathered}
\alpha_{1}, \ldots, \alpha_{r-1}, \beta_{1}, \ldots, \beta_{r-1}, \\
\widetilde{\alpha}_{1}, \ldots, \widetilde{\alpha}_{p}, \widetilde{\alpha}_{1}^{\sigma}, \ldots, \widetilde{\alpha}_{p}^{\sigma}, \widetilde{\beta}_{1}, \ldots, \widetilde{\beta}_{p}, \widetilde{\beta}_{1}^{\sigma}, \ldots, \widetilde{\beta}_{p}^{\sigma}
\end{gathered}
$$

avec donc $\alpha_{i}^{\sigma}=\alpha_{i}$ (invariant), $\beta_{i}^{\sigma}=-\beta_{i}$ (anti-invariant) et $\widetilde{\alpha}_{i}, \widetilde{\beta}_{i}$ entièrement tracés

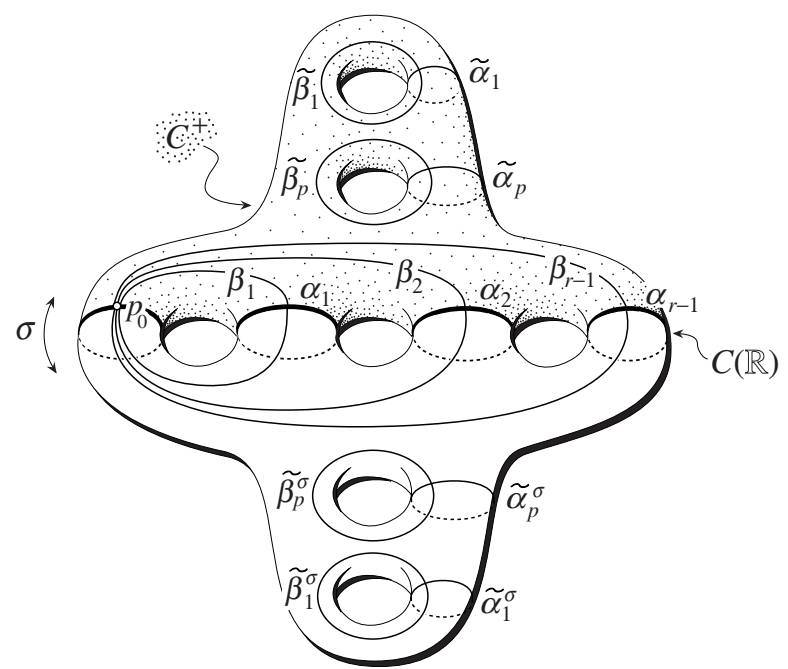

Figure 4. Modèle topologique d'une courbe réelle séparante.

dans la moitié $C^{+}$, et où $p=\frac{g+1-r}{2}$, dont l'intégralité est assurée par la congruence de Klein $r \equiv g+1(\bmod 2)(\mathrm{cf} . \mathrm{p}$. ex. [Gab], p. 142). L'entier $p$ s'interprète comme le genre d'une moitié. Les périodes correspondantes notées

$$
\begin{gathered}
A_{1}, \ldots, A_{r-1}, B_{1}, \ldots, B_{r-1} \\
\widetilde{A}_{1}, \ldots, \widetilde{A}_{p}, \widetilde{A}_{1}^{\sigma}, \ldots, \widetilde{A}_{p}^{\sigma}, \widetilde{B}_{1}, \ldots, \widetilde{B}_{p}, \widetilde{B}_{1}^{\sigma}, \ldots, \widetilde{B}_{p}^{\sigma}
\end{gathered}
$$


satisfont donc $A_{i} \in \mathbb{R}^{g}$ et $B_{i} \in(i \mathbb{R})^{g}$. Il est commode de les visualiser sur la Figure 5 .
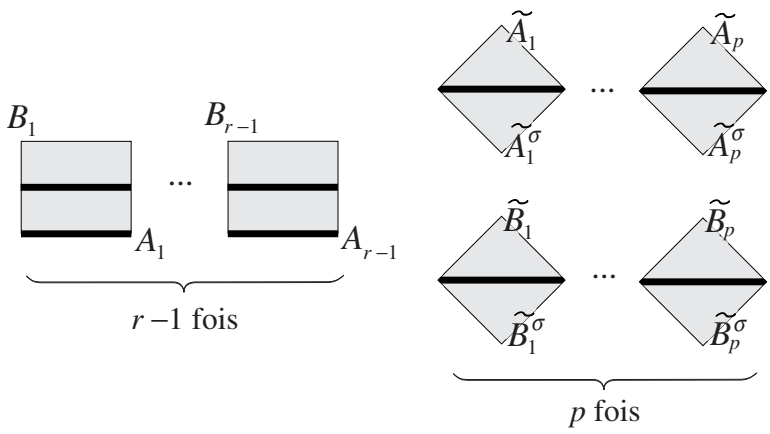

Figure 5. Visualisation de la jacobienne d'une courbe réelle séparante.

Notons que l'involution $\sigma$ sur $J$ préserve les sous-tores $T_{i}$ de dimension 2, définis comme les images par la projection $p: \mathbb{C}^{g} \rightarrow J=\mathbb{C}^{g} / \Lambda$ des 2-plans vectoriels suivants

- $\mathbb{R} A_{i}+\mathbb{R} B_{i}$, pour lesquels $\sigma_{i}$ (= restriction de $\sigma$ à $T_{i}$ ) fixe deux composantes, et

- $\mathbb{R} \widetilde{A}_{i}+\mathbb{R} \widetilde{A}_{i}^{\sigma}, \mathbb{R} \widetilde{B}_{i}+\mathbb{R} \widetilde{B}_{i}^{\sigma}$, où $\sigma_{i}$ fixe une composante.

Dans les deux cas le quotient $T_{i} / \operatorname{Fix}\left(\sigma_{i}\right)$ modulo les points fixes est un cercle, mais dans le premier cas, la classe fondamentale du quotient provient de l'image d'une demi-période, alors que dans le second c'est l'image d'une période complète. Il s'ensuit que les images par l'application $\pi \circ \alpha: C \rightarrow J \rightarrow T^{g}$

- des $r-1$ demi-cycles $\beta_{1}^{+}, \ldots, \beta_{r-1}^{+}$définis par $\beta_{i}^{+}=\beta_{i} \cap \overline{C^{+}}$, i.e. la trace des $\beta_{i}$ sur l'adhérence de $C^{+}$,

- ainsi que des $2 p$ cycles $\widetilde{\alpha}_{1}, \ldots, \widetilde{\alpha}_{p}, \widetilde{\beta}_{1}, \ldots, \widetilde{\beta}_{p}$ donnent $g$ cycles $^{3}$ tracés sur $\Omega \cup\{0\}$ (notons les $\gamma_{1}, \ldots, \gamma_{g}$ ) - qui en outre forment une base de l'homologie du quotient $T^{g}$. D'où le renseignement annoncé.

Tout va découler du lemme suivant, qui de manière imagée affirme que si on irrigue un tore topologiquement, on l'engendre algébriquement (en tant que groupe) :

Lemme 6.1 (Principe d'irrigation). Soit $T^{g}$ un tore de dimension $g$ sur lequel sont tracés g 1-cycles $\gamma_{1}, \ldots, \gamma_{g}: I=[0,1] \rightarrow T^{g}$ qui forment une base de l'homologie de $T^{g}$ (ou plus généralement une famille libre). Alors tout point de $T^{g}$ peut s'écrire comme somme de $g$ points $\pi_{1}+\cdots+\pi_{g}$ avec $\pi_{i} \in \gamma_{i}(I)$ pour $i=1, \ldots, g$.

\footnotetext{
${ }^{3}$ En effet, bien que $\beta_{i}^{+}$ne soit pas un cycle, ses extrémités étant réelles, elles se soudent lorsqu'on quotiente par $J(\mathbb{R}) ;$ c'est pourquoi son image dans $T^{g}$ forme bien un cycle.
} 
Preuve. Il revient au même de voir que l'application $c: I^{g} \rightarrow T^{g}$ définie par $c\left(t_{1}, \ldots, t_{g}\right)=\gamma_{1}\left(t_{1}\right)+\cdots+\gamma_{g}\left(t_{g}\right)$ est surjective. Or $c$ passe au quotient modulo la relation qui identifie les points opposés du cube $I^{g}$ (i.e. ceux dont la différence est dans $\mathbb{Z}^{g}$ ) et donne $\gamma: T^{g} \rightarrow T^{g}$. Il suffit de montrer que le degré topologique de $\gamma$ est non nul. En utilisant le revêtement universel $p: \mathbb{R}^{g} \rightarrow T^{g}=\mathbb{R}^{g} / \mathbb{Z}^{g}$ on montre d'abord que $\gamma$ est homotope à un morphisme de groupes de Lie. En effet soit $\tilde{\gamma_{i}}$ un relevé de $\gamma_{i}$. L'application $\tilde{c}: I^{g} \rightarrow \mathbb{R}^{g}$ définie par $\tilde{c}\left(t_{1}, \ldots, t_{g}\right)=\sum_{i=1}^{g} \tilde{\gamma}_{i}\left(t_{i}\right)$ relève $c$. Comme chacunes des différences $\widetilde{\gamma_{i}}(1)-\widetilde{\gamma_{i}}(0)$ est un vecteur intégral $\xi_{i} \in \mathbb{Z}^{g}$, l'application $s: I^{g} \rightarrow \mathbb{R}^{g}$ définie par $s\left(t_{1}, \ldots, t_{g}\right)=\sum_{i=1}^{g} t_{i} \xi_{i}$ ainsi que l'homotopie canonique $h$ reliant $\tilde{c}$ à $s$, après composition avec $p$, passent au quotient et donnent respectivement $\sigma: T^{g} \rightarrow T^{g}$ un morphisme de groupes de Lie et $H$ une homotopie reliant $\gamma$ à $\sigma$. On conclut alors

- soit en notant que le degré d'un tel morphisme n'est autre que le déterminant de son relevé aux revêtements universels. Ce dernier est non nul puisque via la correspondance canonique $\mathbb{Z}^{g} \simeq H_{1}\left(T^{g}\right)$ les $\xi_{i}$ correspondent aux $\left[\gamma_{i}\right]$.

- Ou bien en utilisant la naturalité (vis-à-vis des morphismes de groupes de Lie) de l'isomorphisme $\wedge^{g} H_{1}\left(T^{g}\right) \rightarrow H_{g}\left(T^{g}\right)$ induit par $H_{1}\left(T^{g}\right) \times \cdots \times H_{1}\left(T^{g}\right) \rightarrow$ $H_{g}\left(T^{g} \times \cdots \times T^{g}\right) \rightarrow H_{g}\left(T^{g}\right)$ où la première flèche est donnée par le produit extérieur en homologie, et la seconde induite par l'addition de $T^{g}$.

Ceci permet de conclure de la façon suivante : on choisit $\pi_{g+1}$ un point de $\Omega$. Noter que $\pi_{g+1}$ est non nul, car $\Omega$ ne contient pas 0 , puisque $\alpha\left(C^{+}\right)$ne rencontre pas $J(\mathbb{R})$ compte tenu du fait que $\alpha: C \rightarrow J$ est un plongement (dès que $g \geq 1$ ) défini sur $\mathbb{R}$. Le Lemme 6.1 appliqué à l'opposé $-\pi_{g+1} \in T^{g}$, montre qu'il existe $g$ points $\pi_{1}, \ldots, \pi_{g}$ situés respectivement sur les courbes $\gamma_{1}, \ldots, \gamma_{g}$ et donc dans $\Omega \cup\{0\}$ tels que $\pi_{1}+\cdots+\pi_{g}=-\pi_{g+1}$. Noter que $\pi_{1}, \ldots, \pi_{g}$ sont non tous nuls vu que $\pi_{g+1} \neq 0$. Ainsi $\pi_{1}+\cdots+\pi_{g}+\pi_{g+1}=0$, ce qui exprime bien 0 comme somme de $d \leq g+1$ points qu'on peut supposer être dans $\Omega$, quitte à omettre les $\pi_{i}$ qui seraient éventuellement nuls. D'où l'existence d'un morphisme saturé avec même un contrôle sur son degré; de façon précise nous avons montré le :

Théorème 6.2 (Ahlfors). Soit $C$ une courbe séparante de genre g. Il existe alors un morphisme saturé $f: C \rightarrow \mathbb{P}^{1}$. De plus il est toujours possible d'exhiber un tel morphisme de degré inférieur ou égal à $g+1$, mais le degré de $f$ ne peut être abaisser en dessous du nombre $r$ de composantes connexes de la partie réelle, qui constitue une borne inférieure absolue pour le degré d'un morphisme saturé.

Remarque 6.3. L'exemple de la Gürtelkurve $C_{4}$ montre qu'on ne peut pas toujours escompter trouver un morphisme saturé qui réalise la borne inférieure $r$. En effet du fait que le nombre minimal de feuillets requis pour concrétiser une courbe plane lisse $C_{m} \subset \mathbb{P}^{2}$ de degré $m$ comme revêtement de $\mathbb{P}^{1}$ est $m-1$, la Gürtelkurve est seulement trigonale. Il est donc impossible d'atteindre $r=2$. 
Mise en garde 6.4. Il ne faut pas croire que $g+1$ constitue une borne supérieure pour le degré d'un morphisme saturé, comme pourrait le laisser penser l'ambigüité de la formulation suivante extraite de l'encyclopédie japonaise (cf. [EDM], p. 1367) :

L. Ahlfors proved that a Riemann surface of genus $g$ bounded by $m$ contours can be mapped conformally to an at most $(2 g+m)$-sheeted unbounded covering surface of the unit disk.

En effet une fois l'existence d'un diviseur unilatéral $D$ tel que $D \sim D^{\sigma}$ établie, on peut évidemment considérer ses multiples $k D$ qui satisferont encore la condition d'unilatéralité, ainsi que $k D \sim k D^{\sigma}$ et qui induiront donc des morphismes saturés de degré $k \operatorname{deg}(D)$, que l'on peut rendre arbitrairement grand.

\section{Amélioration quantitative du théorème d'Ahlfors}

Dans son aspect quantitatif le Théorème 6.2 coïncide exactement avec le résultat d'Ahlfors (cf. [Ah], Theorem 10, p. 132). Noter que ce degré de concrétisation tient seulement compte du genre $g$, mais ignore l'invariant $r$. On propose dans ce qui suit de montrer que le résultat d'Ahlfors peut être substantiellement amélioré, en représentant la surface avec un nombre plus économique (mais néanmoins universel, c'est-à-dire qui dépend seulement de la topologie et non de la structure complexe) de feuillets au-dessus du disque, et ce en tenant parfaitement compte de la topologie de la surface de Riemann à bord. Evidemment, compte tenu de la Remarque 3.2, le résultat d'Ahlfors est optimal pour les courbes Harnack-maximales avec $r=g+1$ qui correspondent aux surfaces de Riemann à bord planes ${ }^{4}$, c'est-à-dire celles qui se plongent dans le plan complexe $\mathbb{C}$. Mais par contre on va voir qu'il n'en est rien en général, en montrant qu'il est toujours possible d'exhiber un morphisme saturé de degré égal à la moyenne de $r$ et $g+1$. De façon précise :

Théorème 7.1. Soit $C$ une courbe séparante de genre g etr le nombre de composantes de $C(\mathbb{R})$. Il existe alors toujours un morphisme saturé $f: C \rightarrow \mathbb{P}^{1}$ de degré inférieur ou égal à $\frac{r+g+1}{2}=r+p$ ( $p$ s'interprétant alors comme le genre d'une moitié de $C$ ).

Enoncé en termes de surfaces de Riemann à bord cela devient :

Théorème 7.2. Soit $S$ une surface de Riemann à bord compacte de genre $p$ avec $r$ composantes de bord. Il existe alors toujours (indépendamment de la structure analytique complexe sur $S$ ) un revêtement holomorphe (ramifié) vers le disque $f: S \rightarrow$ $\Delta=\{z \in \mathbb{C}|| z \mid \leq 1\}$ de degré inférieur ou égal à $r+p=\frac{r+g+1}{2}$ ( $g$ s'interprétant alors comme le genre du double de $S$ ).

\footnotetext{
${ }^{4}$ Classiquement qualifiées de schlicht, puisqu'en allemand "schlichten" signifie "aplanir".
} 
Exemple 7.3. Pour illustrer ce nouveau théorème considérons encore l'exemple de la Gürtelkurve. Le Théorème 7.1 prédit l'existence d'un morphisme saturé de degré égal à la moyenne de $r=2$ et $g+1=3+1=4$, soit $d=3$. Tel est bien le cas comme on le voit en laissant dégénérer le centre de projection sur l'ovale interne de la quartique (cf. Figure 6).

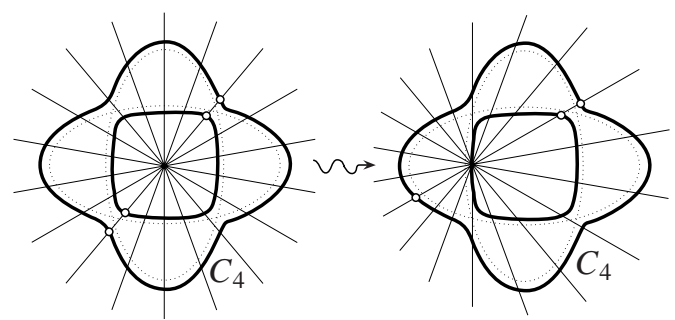

Figure 6

Un autre exemple plus sophistiqué est le suivant : on considère la sextique $C_{6}$ obtenue en déformant la configuration suivante de trois coniques (cf. Figure 7). Les lis-

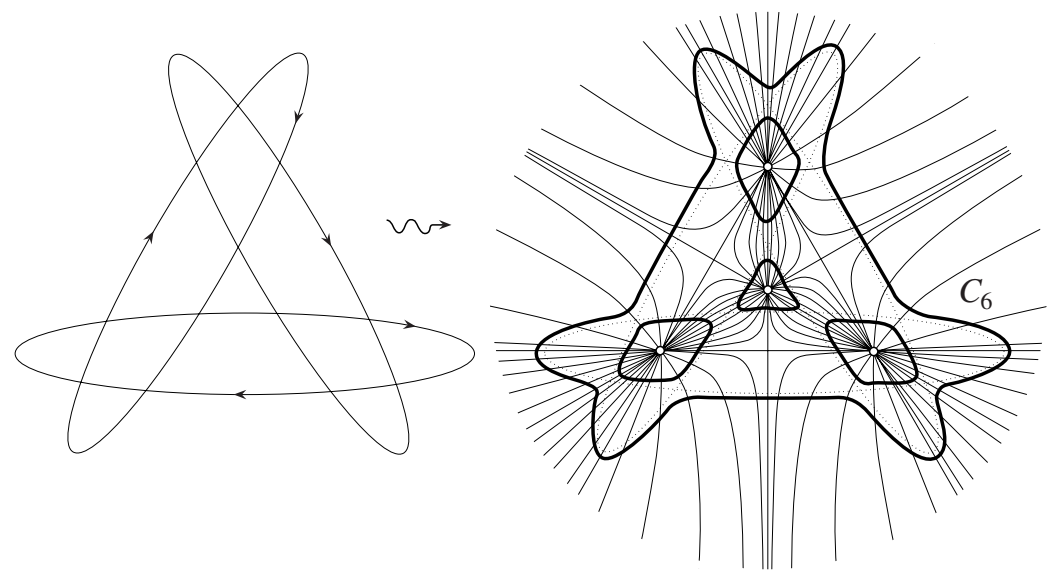

Figure 7

sifications effectuées étant compatibles avec les orientations, on sait à priori (d'après un résultat de Fiedler, cf. [Fi]) que cette courbe est séparante. Mais cela peut aussi se voir de façon synthétique en considérant le pinceau des coniques passant par 4 points distribués à l'intérieur des 4 ovales les plus profonds. Toutes les coniques de ce pinceau découpent 12 points réels sur $C_{6}$, ce qui redémontre le caractère séparant de $C_{6}$. Le morphisme saturé est ici de degré 12 . Or on s'attend avec 7.1 à ce qu'il en existe 
un de degré la moyenne de $r=5$ et $g+1=11$, i.e. 8 et tel est bien le cas comme on le voit en laissant à nouveau dégénérer les 4 centres de projection sur les ovales.

Avant d'entreprendre la démonstration de ce nouveau résultat, je tiens à exposer le cheminement qui m'a suggéré la possibilité d'une telle amélioration. C'est l'objet du paragraphe suivant.

\section{Excursion chez les courbes complexes et justification heuristique du théorème d'existence raffiné}

Il est instructif de voir ce que donne la méthode d'irrigation dans le contexte plus "simple" d'une courbe algébrique complexe $C$ (lisse irréductible). [Plus simple, du fait que la topologie d'une telle courbe est épinglée par un seul entier le genre $g$, alors qu'il en faut deux $g$ et $r$ pour spécifier celle d'une courbe réelle séparante.] Comme le morphisme d'Abel $\alpha: C \rightarrow J$ induit un isomorphisme sur le $H_{1}$, le principe d'irrigation s'applique aux images d'une base de $H_{1}(C, \mathbb{Z})$, et montre que tout point de la jacobienne peut s'écrire comme somme de $2 g$ points qui coulissent sur les $2 g$ courbes fondamentales. Cela donne la surjectivité de $\alpha: C^{(d)} \rightarrow J\left[C^{(d)}\right.$ désignant la variété des groupes de $d$ points de la courbe] pour $d=2 g$, alors que la surjectivité vaut déjà pour $d=g$ (d'après le classique théorème d'inversion de Jacobi). Cette défaillance de notre méthode dans le cas classique d'une surface de Riemann fermée suggère la possibilité d'améliorer le théorème d'Ahlfors. En outre cette défaillance n'est qu'apparente, pour la raison suivante :

La courbe $C$ est plongée dans sa jacobienne $J$ qui est un tore de dimension $2 g$, et cette courbe est une surface orientable de genre $g$, mais modulo une homologie elle y est plongée comme $g$ tores de dimension 2 qui irriguent le tore $J=T^{2 g}$. Par suite tout point de $J$ peut s'écrire comme somme de $g$ points situés sur ces $g$ tores. En espérant que cette propriété se conserve lors d'une "homologie", on trouve une justification heuristique du théorème d'inversion de Jacobi. On reconnaît sans peine qu'il ne s'agit ici que d'un déguisement du principe d'irrigation : à l'irrigation unidimensionnel (à laquelle nous nous étions initialement limité) se substitue maintenant une irrigation par des cycles bidimensionnels.

Cette observation étant faite, revenons à notre problème sur les courbes réelles séparantes. La prédiction analogue conduit au Théorème 7.1. En effet pour une courbe séparante, le système constitué

- des $r-1$ cycles $B_{1}^{+}, \ldots, B_{r-1}^{+}$définies comme les images des $\beta_{i}^{+}$- qui rappelons-le sont les traces des cycles $\beta_{i}$ sur l'adhérence de la moitié (cf. Figure 4 ou 8) - dans le tore quotient $T^{g}=J / J(\mathbb{R})$,

- et des $p$ tores $\tau_{1}, \ldots, \tau_{p}$ bidimensionnels définis pour l'instant seulement intuitivement au moyen de la Figure 8 qui sont tous "dessinables" sur la moitié - 

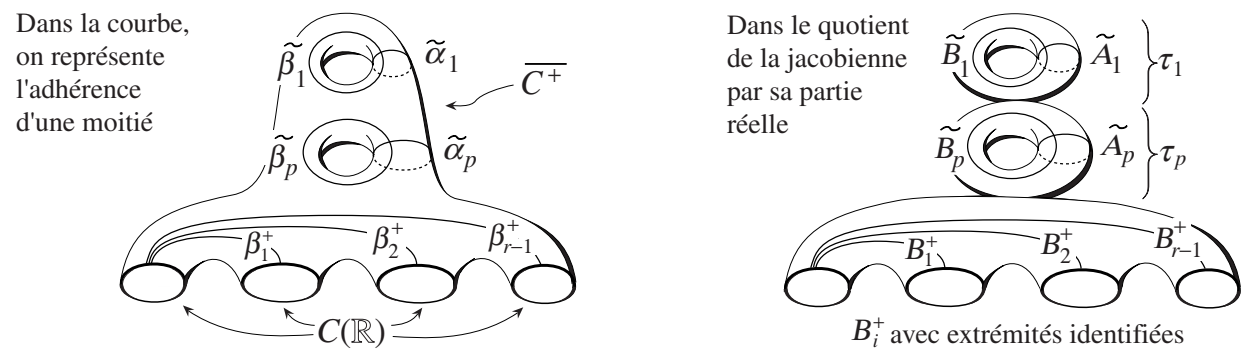

Figure 8

irrigue le tore quotient $T^{g}$, de sorte que 0 peut être exprimé comme somme de $(r-1)+p+1=r+p$ points de $\Omega$, d'où une justification heuristique du Théorème 7.1.

La suite de l'article n'est qu'une formalisation des deux arguments heuristiques que nous venons de donner. L'intérêt de formaliser aussi l'inversion de Jacobi est qu'elle offre un terrain plus propice pour un premier exercice de formalisation de l'idée de l'irrigation. Cette expérience acquise, on affrontera avec plus d'aisance la situation plus touffue émanant d'une courbe réelle séparante.

\section{Preuve topologique du théorème d'inversion de Jacobi}

On considère le morphisme d'Abel $\alpha: C^{g} \rightarrow J$ défini cette fois au niveau du produit $C^{g}$ et il suffit pour établir sa surjectivité, de montrer que son degré topologique est non nul. Il s'agit d'étudier l'homomorphisme

$$
H_{2 g}(\alpha): H_{2 g}\left(C^{g}\right) \rightarrow H_{2 g}(J)
$$

induit en homologie de dimension maximale. L'idée est d'exploiter la structure de groupe sur $J$ pour se ramener à savoir ce qu' induit $\alpha: C \rightarrow J$ au niveau du $H_{2}$. Ceci motive l'introduction du formalisme suivant :

Pour un couple d'entiers $p, q$ on dispose du produit extérieur en homologie $H_{p}(J) \times H_{q}(J) \stackrel{\times}{\longrightarrow} H_{p+q}(J \times J)$, qui composé avec $H_{p+q}(J \times J) \rightarrow H_{p+q}(J)$ l'homomorphisme induit par l'addition de $J$, fournit une opération bilinéaire

$$
\star: H_{p}(J) \times H_{q}(J) \longrightarrow H_{p+q}(J)
$$

appelée le produit de Pontrjagin (cf. [Pon] ou [LB], p. 104). On peut plus généralement le définir pour tout groupe de Lie $G$ (voire un groupe topologique). Cependant dans le cas abélien, il satisfait en outre à la relation d'anticommutativité : $\sigma \star \tau=(-1)^{p q} \tau \star \sigma$ 
pour $\sigma$ et $\tau$ des classes de dimensions respectives $p$ et $q$ (cf. [Do], p. 195). Il suit de là que l'anneau de Pontrjagin $\left(H_{*}\left(T^{n}\right),+, \star\right)$ d'un tore $T^{n}$ (de dimension réelle $n$ ) est isomorphe - en tant qu'algèbre graduée - à l'algèbre extérieure sur sa composante homogène de degré un $H_{1}\left(T^{n}\right): H_{*}\left(T^{n}\right) \cong \wedge H_{1}\left(T^{n}\right)$. En particulier si $\gamma_{1}, \cdots, \gamma_{n}$ est une base de $H_{1}\left(T^{n}\right)$, le produit de Pontrjagin $\gamma_{1} \star \cdots \star \gamma_{n}$ décrit une classe fondamentale de $T^{n}$.

On a alors le diagramme commutatif :

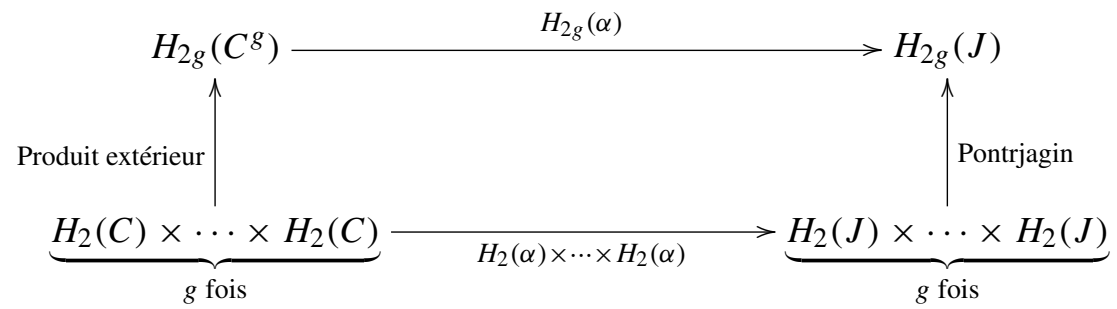

dont la commutativité provient de la naturalité du produit extérieur homologique, puisque $\alpha: C^{g} \rightarrow J$ n'est autre que la composée $C \times \cdots \times C \rightarrow J \times \cdots \times J \rightarrow J$ du produit de $\alpha: C \rightarrow J g$-fois par lui-même avec l'addition de $J$. La connaissance de $H_{2 g}(\alpha)$ se ramène donc à celle de $H_{2}(\alpha)$; qui fait l'objet du :

Lemme 9.1. Soit $\alpha_{1}, \ldots, \alpha_{g}, \beta_{1}, \ldots, \beta_{g}$ une base symplectique de $H_{1}(C, \mathbb{Z})$, c'està-dire telle que $\alpha_{i} \cdot \alpha_{j}=\beta_{i} \cdot \beta_{j}=0$ et $\alpha_{i} \cdot \beta_{j}=\delta_{i j}$, et notons $A_{1}, \ldots, A_{g}, B_{1}, \ldots, B_{g}$ leurs images dans $H_{1}(J, \mathbb{Z})$ par le morphisme d'Abel $\alpha: C \rightarrow J$. Alors l'image par $H_{2}(\alpha)$ de la classe fondamentale $[C]$ de $C$ est $\sum_{i=1}^{g} A_{i} \star B_{i}$.

Preuve. L'idée est qu'après avoir contracté la surface de genre $g$ sous-jacente à $C$, sur un bouquet $B$ de $g$ tores de dimension 2 comme l'indique la Figure 9, on peut trouver une factorisation "homotopique"

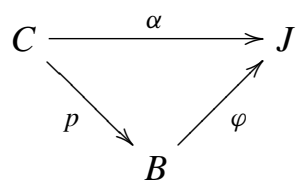

du morphisme d'Abel $\alpha$ au travers de ce bouquet, i.e. une application $\varphi$ qui fasse commuter le diagramme (2) à homotopie près. Pour l'application composée $\varphi \circ p$, il sera alors flagrant qu'elle enverra la classe fondamentale de $C \operatorname{sur} \sum_{i=1}^{g} A_{i} \star B_{i}$, d'où le lemme par invariance homotopique de l'homologie. L'argument détaillé est le suivant :

Préliminaire sur le modèle $4 g$-gonale. On représente d'abord la surface de genre $g$, sous-jacente à $C$, de la façon habituelle : soit comme quotient d'un $4 g$-gone $\Delta$ [homéomorphe au disque] modulo la relation d'équivalence, qui identifie les points du 


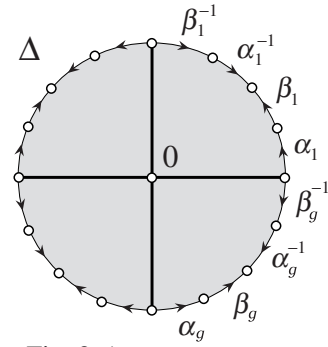

Fig. 9.a)

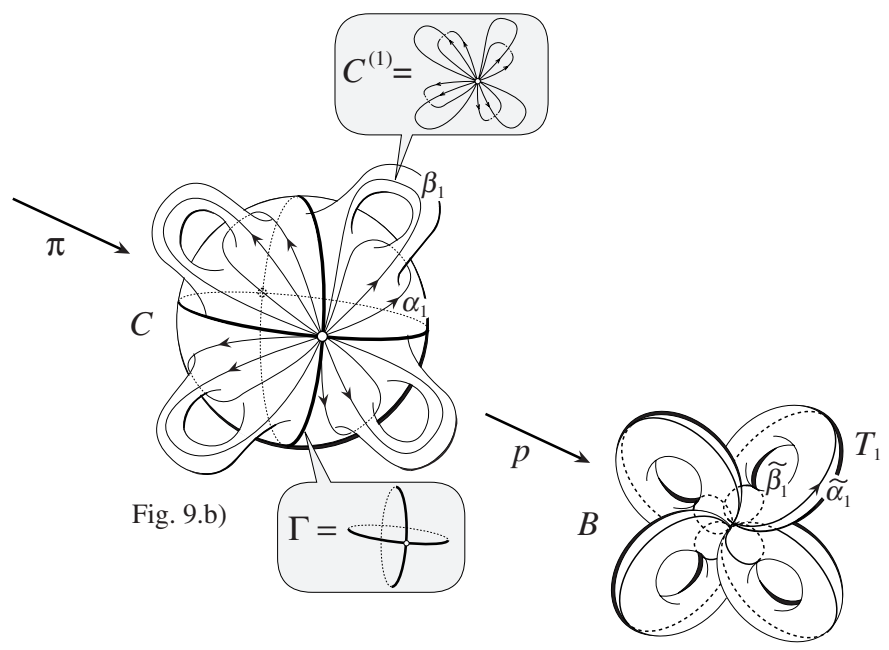

Figure 9

bord de $\Delta$ suivant le code $\alpha_{1} \beta_{1} \alpha_{1}^{-1} \beta_{1}^{-1} \ldots \alpha_{g} \beta_{g} \alpha_{g}^{-1} \beta_{g}^{-1}$ (cf. Figure 9.a)). On note $C^{(1)}$ l'image du bord $\partial \Delta$ dans $C$, puisque ce n'est autre que le 1-squelette de $C$, pour la structure cellulaire introduite par cette représentation $4 g$-gonale. Noter que c'est ici qu'on fait usage de l'hypothèse symplectique, faite sur la base du $H_{1}$. En effet la représentation $4 g$-gonale livre non seulement la surface mais aussi une base de son $H_{1}$ en orientant le 1-squelette comme bord de la 2-cellule, base qui est symplectique. Inversement étant donné une base symplectique, on peut toujours la représenter de la sorte.

Contraction du bretzel $C$ sur le bouquet $B$. En quotientant $C$ par le sous-espace $\Gamma$ défini comme l'image par la projection $\pi: \Delta \rightarrow C$ des $g$ rayons issus de l'origine 0 du disque $\Delta$, et reliant 1 sommet sur 4 (les origines des $\alpha_{i}$ ) du $4 g$-gone $\Delta$ (cf. Figure 9.a)), on obtient un bouquet $B=\bigvee_{i=1}^{g} T_{i}$ de $g$ tores $T_{i}$ de dimension 2, vu qu'une fois ces $g$ rayons contractés on voit $g$ 2-cellules munies chacune de la relation d'équivalence $\alpha_{i} \beta_{i} \alpha_{i}^{-1} \beta_{i}^{-1}$ sur leur bord.

Construction de l'application $\varphi$. Evidemment $\alpha: C \rightarrow J$ n'étant pas constante sur $\Gamma$ (car injective dès que $g \geq 1$ ), elle ne passe pas au quotient $B=C / \Gamma$. Par contre sa restriction à $C^{(1)}$ induit du fait que $C^{(1)}$ ne rencontre $\Gamma$ qu'en un seul point, une application $\varphi^{(1)}: B^{(1)} \rightarrow J$ définie sur $B^{(1)}$ l'image de $C^{(1)}$ dans $B$. Comme le tore $T_{i}$ s'identifie au produit $\widetilde{\alpha}_{i} \times \widetilde{\beta}_{i}$ des images $\widetilde{\alpha}_{i}, \widetilde{\beta}_{i}$ de $\alpha_{i}, \beta_{i}$ dans le bouquet $B$, on peut définir $\varphi_{i}: T_{i} \rightarrow J$ comme la composée du produit $\varphi^{(1)}{ }_{\mid \widetilde{\alpha}_{i}} \times \varphi^{(1)}{ }_{\mid \widetilde{\beta}_{i}}: T_{i} \rightarrow J \times J$ avec l'addition de $J$. On obtient $\varphi$ en recollant les $\varphi_{i}$. 
Vérification du fait flagrant. L'application $\widetilde{\alpha}: C \rightarrow J$ composée de $p$ avec $\varphi$ satisfait alors $H_{2}(\widetilde{\alpha})([C])=\sum_{i=1}^{g} A_{i} \star B_{i}$. En effet [C] s'envoie d'abord - via $H_{2}(p)-$ sur la somme $\sum_{i=1}^{g}\left[T_{i}\right]$ des classes fondamentales $\left[T_{i}\right]$ des tores $T_{i}$. Or par construction de $\varphi$, on a $H_{2}(\varphi)\left(\left[T_{i}\right]\right)=A_{i} \star B_{i}$ (résulte de la naturalité du produit extérieur). Par suite il vient bien $H_{2}(\widetilde{\alpha})([C])=H_{2}(\varphi \circ p)([C])=H_{2}(\varphi)\left(H_{2}(p)([C])\right)=$ $H_{2}(\varphi)\left(\sum_{i=1}^{g}\left[T_{i}\right]\right)=\sum_{i=1}^{g} H_{2}(\varphi)\left(\left[T_{i}\right]\right)=\sum_{i=1}^{g} A_{i} \star B_{i}$.

Commutativité du triangle (2) à homotopie près. Il reste à vérifier que les applications $\alpha$ et $\tilde{\alpha}$ sont homotopes. Mais c'est là une simple conséquence du fait qu'elles ont même restriction au 1-squelette de $C$. En effet, de ce fait on peut définir une application continue $F: \partial(\Delta \times I)=(\Delta \times \partial I) \cup(\partial \Delta \times I) \rightarrow J$ en recollant les applications suivantes :

- $\alpha \circ \pi$ sur la base inférieure $\Delta \times\{0\}$ du "cylindre plein" $\Delta \times I$,

- $\widetilde{\alpha} \circ \pi$ sur sa base supérieure $\Delta \times\{1\}$,

- et $\alpha \circ \pi \mid \partial \Delta: \partial \Delta \rightarrow J$ sur le cylindre $\partial \Delta \times I$ identiquement au cours du temps.

On dispose à ce stade de $F$ définie sur le bord $\partial(\Delta \times I)$ de la 3-cellule $\Delta \times I$, bord qui est homéomorphe à une sphère $S^{2}$. Or $J \approx T^{2 g}$ étant homéomorphe à un tore, on déduit - du fait que $\left[S^{2}, T^{n}\right]=\{*\}$ ne contient à homotopie près que l'application constante - que $F$ s'étend à la 3-cellule $\Delta \times I$. Extension qui fournit (par passage au quotient de $\Delta$ à $C$ ) l'homotopie cherchée reliant $\alpha$ à $\widetilde{\alpha}$.

On est maintenant en mesure de conclure. La commutativité du diagramme (1) jointe au Lemme 9.1 montre que la classe fondamentale du produit $C^{g}$ qui n'est autre que le produit de la classe fondamentale de $C$, s'envoie via $H_{2 g}(\alpha)$ sur

$$
\left(\sum_{i=1}^{g} A_{i} \star B_{i}\right) \star \cdots \star\left(\sum_{i=1}^{g} A_{i} \star B_{i}\right) .
$$

Posons $\tau_{i}=A_{i} \star B_{i}$. La relation d'anticommutativité montre que $\tau_{i} \star \tau_{i}=0$, de sorte que les seules contributions proviennent des facteurs $\tau_{i_{1}} \star \cdots \star \tau_{i_{g}}$ avec $i_{j}$ distincts deux à deux. Comme les $\tau_{i}$ sont de dimension 2 , ils commutent entre eux si bien que l'expression (3) se réduit - après réordonnage - à $g !\left(\tau_{1} \star \cdots \star \tau_{g}\right)=g ![J]$. La dernière égalité repose sur le fait que pour un tore $T^{n}$ de dimension $n$ le produit de Pontrjagin $\gamma_{1} \star \cdots \star \gamma_{n}$ d'une base $\gamma_{1}, \ldots \gamma_{n}$ de $H_{1}\left(T^{n}\right)$ représente sa classe fondamentale. Le degré de $\alpha: C^{g} \rightarrow J$ est donc $g ! \neq 0$, d'où la surjectivité cherchée de $\alpha: C^{g} \rightarrow J$.

Note. Une approche purement topologique au théorème d'inversion de Jacobi est aussi présentée dans le livre de Clemens (cf. [Cl], pp. 131-132). 


\section{Démonstration du théorème d'existence raffiné}

On note $\bar{\Omega}=\Omega \cup\{0\}$, où $\Omega$ est l'image de la moitié $C^{+}$dans le tore quotient $T^{g}=$ $J / J(\mathbb{R})$. Pour établir le Théorème 7.1 affirmant qu'il existe toujours un morphisme saturé de degré inférieur ou égal à $r+p$, il suffit (d'après le §6) de montrer l'assertion suivante :

Proposition 10.1. L'ensemble $(d-1) \bar{\Omega}=\left\{\pi_{1}+\cdots+\pi_{d-1} \mid \pi_{i} \in \bar{\Omega}\right\}$ est égal à tout $T^{g}$ pour $d=r+p$.

Preuve. Il s'agit d'établir la surjectivité de l'application $\psi: \bar{\Omega}^{d-1} \rightarrow T^{g}$ donnée par $\left(\pi_{1}, \ldots, \pi_{d-1}\right) \mapsto \sum_{i=1}^{d-1} \pi_{i}$. On va le faire en s'appuyant sur le :

Lemme 10.2. Soit $f: X \rightarrow M$ une application continue d'un espace topologique $X$ dans une variété connexe $M$ de dimension $n$. Si l'homomorphisme induit en homologie $H_{n}(f): H_{n}(X, \mathbb{Z}) \rightarrow H_{n}(M, \mathbb{Z})$ est non nul, alors $f$ est surjective.

Preuve. Sinon $f$ évite un point $p$ de $M$. Mais $M-p$ étant une variété connexe (si $n \geq 2$ ) non compacte, on sait que $H_{n}(M-p)=0$ (cf. p. ex. [Vi], p. 152), et lorsque $n \leq 1$ ceci vaut aussi trivialement. Comme $f$ factorise par $M-p$ on en tire par fonctorialité que $H_{n}(f)=0$.

Note. En fait pour notre problème on utilise 10.2 seulement dans le cas particulier où $M$ est un tore, auquel cas la preuve de $H_{n}(M-p)=0$ peut être simplifiée.

D'après Lemme 10.2, il nous suffit de vérifier que $H_{g}(\psi)$ est non nul. Vu que $(r-1)+2 p=g$ et comme $d=r+p$, on a un diagramme commutatif :

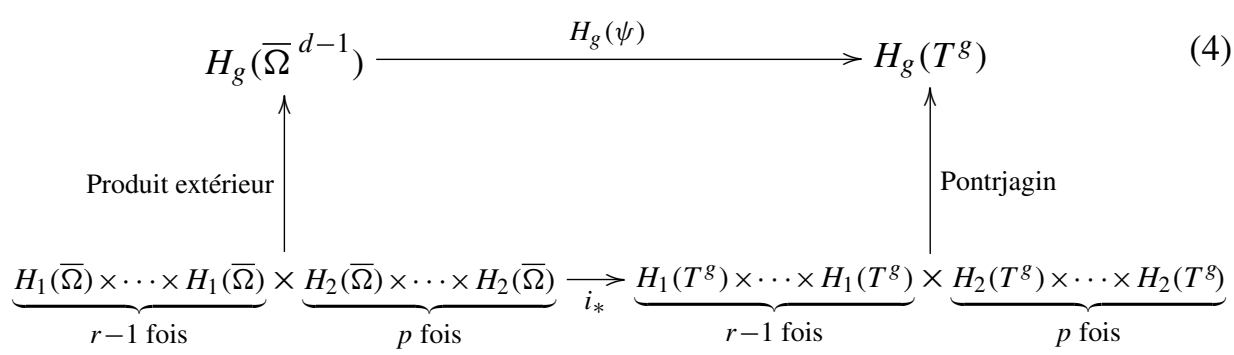

où $i_{*}$ est la flèche induite par l'inclusion $i: \bar{\Omega} \hookrightarrow T^{g}$. La connaissance de $H_{g}(\psi)$ se ramène donc à celle de $H_{2}(i): H_{2}(\bar{\Omega}) \rightarrow H_{2}\left(T^{g}\right)$, qui est pécisée par le :

Lemme 10.3. Il existe un élément $\omega \in H_{2}(\bar{\Omega})$ (essentiellement la "classe fondamentale" de $\bar{\Omega})$ dont l'image par $H_{2}(i)$ est $\sum_{i=1}^{p} \widetilde{A}_{i} \star \widetilde{B}_{i}$, où $\widetilde{A}_{i}, \widetilde{B}_{i}$ sont les images dans $T^{g}$ des $\widetilde{\alpha}_{i}, \widetilde{\beta}_{i}$ définis par la Figure 4. 

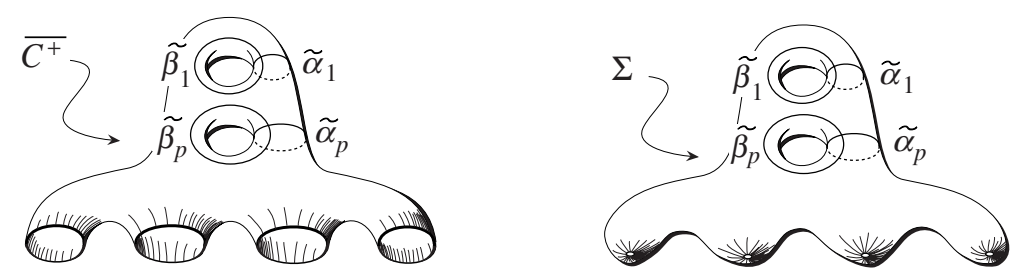

Figure 10

Preuve. Le quotient de la surface à bord $\overline{C^{+}}$par la relation d'appartenance à une même composante de bord est une surface $\Sigma$ de genre $p$ (cf. Figure 10). La composée $\overline{C^{+}} \stackrel{\alpha}{\rightarrow} J \rightarrow T^{g}$ passe au quotient et donne $\varepsilon: \Sigma \rightarrow \bar{\Omega}$ (lorsque restreinte à son image); qui suivie de l'inclusion $\bar{\Omega} \stackrel{i}{\hookrightarrow} T^{g}$ donne $\beta: \Sigma \stackrel{\varepsilon}{\rightarrow} \bar{\Omega} \stackrel{i}{\hookrightarrow} T^{g}$, qui va $d^{\prime} u n$ bretzel vers un tore : donc un analogue du morphisme d'Abel $\alpha: C \rightarrow J$. On peut alors recopier la preuve du Lemme 9.1, et conclure à l'existence d'une factorisation homotopique de $\beta$ au travers d'un bouquet $B$ de $p$ tores

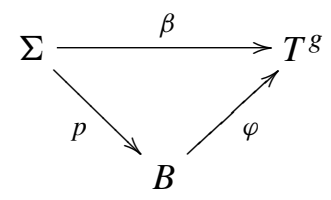

et cela conduit à la formule $H_{2}(\beta)([\Sigma])=\sum_{i=1}^{p} \widetilde{A}_{i} \star \widetilde{B}_{i}$. Or il est naturel de définir la classe fondamentale $\omega$ de $\bar{\Omega}$ comme $H_{2}(\varepsilon)([\Sigma])$, et alors $H_{2}(i)(\omega)=$ $H_{2}(i)\left(H_{2}(\varepsilon)([\Sigma])\right)=H_{2}(i \circ \varepsilon)([\Sigma])=H_{2}(\beta)([\Sigma])=\sum_{i=1}^{p} \widetilde{A}_{i} \star \widetilde{B}_{i}$. D'où le lemme.

Vérifions maintenant que $H_{g}(\psi)$ est non trivial. En effet, évaluons le sur l'élément $b_{1}^{+} \times \cdots \times b_{r-1}^{+} \times \omega \times \cdots \times \omega(p$ fois $\omega)$, où $b_{i}^{+} \in H_{1}(\bar{\Omega})$ est la classe d'homologie définie par le cycle obtenu en projetant dans $T^{g}$ le chemin $\beta_{i}^{+}$défini par la Figure 4. On obtient compte tenu de la commutativité du diagramme (4) et du Lemme 10.3 :

$$
\begin{aligned}
H_{g}(\psi)\left(b_{1}^{+}\right. & \left.\times \cdots \times b_{r-1}^{+} \times \omega \times \cdots \times \omega\right) \\
& =H_{1}(i)\left(b_{1}^{+}\right) \star \cdots \star H_{1}(i)\left(b_{r-1}^{+}\right) \star H_{2}(i)(\omega) \star \cdots \star H_{2}(i)(\omega) \\
& =B_{1}^{+} \star \cdots \star B_{r-1}^{+} \star\left(\sum_{i=1}^{p} \widetilde{A}_{i} \star \widetilde{B}_{i}\right) \star \cdots \star\left(\sum_{i=1}^{p} \widetilde{A}_{i} \star \widetilde{B}_{i}\right),
\end{aligned}
$$

où $B_{i}^{+}$désigne l'image de $b_{i}^{+}$par $H_{1}(i)$. Posons $\tau_{i}=\widetilde{A}_{i} \star \widetilde{B}_{i}$. Comme $\tau_{i} \star \tau_{i}=0$, les seules termes qui subsistent, s'obtiennent en prenant de chacune des $p$ sommes 
des $\tau_{i}$ distincts deux à deux. L'expression ci-dessus se réduit donc - du fait que les $\tau_{i}$ étant de dimension 2 commutent entre eux - à $B_{1}^{+} \star \cdots \star B_{r-1}^{+} \star p !\left(\tau_{1} \star \cdots \star \tau_{p}\right)$. Or la classe fondamentale du but $T^{g}$ est $\left[T^{g}\right]=B_{1}^{+} \star \cdots \star B_{r-1}^{+} \star \tau_{1} \star \cdots \star \tau_{p}$, car d'après le $\S 6$ les $B_{1}^{+}, \ldots, B_{r-1}^{+}, \widetilde{A}_{1}, \widetilde{B}_{1}, \ldots, \widetilde{A}_{p}, \widetilde{B}_{p}$ forment une base de $H_{1}\left(T^{g}\right)$. L'élément envisagé a donc pour image $p !\left[T^{g}\right] \neq 0$, d'où la non-nullité de $H_{g}(\psi)$. Ce qui achève la preuve de la Proposition 10.1.

Remerciements. Ils vont à D. Coray, M. Kervaire, F. Ronga et C. Weber pour leur aide, ainsi qu'à J. Huisman, F. Mangolte et au rapporteur pour l'intérêt et les améliorations qu'ils ont apporté à ce travail.

\section{Références}

[Ah] L. V. Ahlfors, Open Riemann surfaces and extremal problems on compact subregions. Comment. Math. Helv. 24 (1950), 100-134. Zbl 0041.41102 MR 0036318

[AS] L. V. Ahlfors, L. Sario, Riemann Surfaces. Princeton University Press, Princeton, N.J., 1960. Zbl 0196.33801 MR 0114911

[AG] N. L. Alling, N. Greenleaf, Klein surfaces and real algebraic function fields. Bull. Amer. Math. Soc. 75 (1969), 869-872. Zbl 0203.23603 MR 0251213

[Bi] L. Bieberbach, Über einen Riemannschen Satz aus der Lehre von der konformen Abbildung. Sitzungs-Ber. Berliner math. Ges. 24 (1925), 6-9. JFM 51.0273 .02

[Cl] C.H. Clemens, A Scrapbook of Complex Curve Theory. Plenum Press, New York, London 1980. Zbl 0456.14016 MR 0614289

[Do] A. Dold, Lectures on Algebraic Topology. Grundlehren Math. Wiss. 200, SpringerVerlag, Berlin 1972. Zbl 0234.55001 MR 0415602

[EC] F. Enriques, O. Chisini, Lezioni sulla Teoria Geometrica delle Equazioni e delle Funzioni Algebriche. Zanichelli, Bologna 1915-1918. JFM 46.0941.11 MR 0966664

[EDM] Encyclopedic Dictionary of Mathematics. Vol. I-IV, ed. by Kiyosi Itô, 2nd ed., MIT Press, Cambridge, MA, 1987. Zbl 0674.00025 MR 0901762

[Fi] T. Fiedler, Eine Beschränkung für die Lage von reellen ebenen algebraischen Kurven. Beiträge Algebra Geom. 11 (1981), 7-19. Zbl 0505.14041 MR 0680452

[Gab] A. Gabard, Topologie des courbes algébriques réelles : une question de Felix Klein. L'Enseign. Math. 46 (2000), 139-161. Zbl 0986.14040 MR 1769940

[GM] W.-D. Geyer, G. Martens, Überlagerungen berandeter Kleinscher Flächen. Math. Ann. 228 (1977), 101-111. Zbl 0354.14010 MR 0444671

[Gr1] H. Grunsky, Über die konforme Abbildung mehrfach zusammenhängender Bereiche auf mehrblättrige Kreise. Sitzungsber. Preuß. Akad. (1937), 40-46. Zbl 0016.26701

[Gr2] H. Grunsky, Über die konforme Abbildung mehrfach zusammenhängender Bereiche auf mehrblättrige Kreise, II. Abh. Preuß. Akad. Wiss. Math.-nat. Kl. no. 11, (1941) 1-8. Zbl 0026.22001 MR 0018748

[Hui] J. Huisman, On the geometry of algebraic curves having many real components. Rev. Mat. Complut. vol. XIV, num. 1 (2001), 83-92. Zbl 1001.14021 MR 1851723 
[K] F. Klein, Gesammelte mathematische Abhandlungen. Dritter Band, 1922 ; Reprint, Springer-Verlag, Berlin 1973. Zbl 0269.01017 MR 0389520

[LB] H. Lange, Ch. Birkenhake, Complex Abelian Varieties. Grundlehren Math. Wiss. 302, Springer-Verlag, Berlin 1980. Zbl 0779.14012 MR 1217487

[Neh] Z. Nehari, Conformal Mapping of Open Riemann Surfaces. Trans. Amer. Math. Soc. 68 (2) (1950), 258-277. Zbl 0041.41201 MR 0034458

[Pon] L. S. Pontrjagin, Homologies in Compact Lie Groups. Mat. Sbornik, Novaya Seriya 6 (3) (1939), 389-422. Zbl 0022.31602 MR 0001563

[Rie] B. Riemann, Gleichgewicht der Electricität auf Cylindern mit kreisförmigem Querschnitt und parallelen Axen. Conforme Abbildung von durch Kreise begrenzten Figuren (Nachlass XXVI). In Gesammelte mathematische Werke, wissenschaftlicher Nachlass und Nachträge, nach der Ausgabe von H. Weber und R. Dedekind, neu herausgegeben von R. Narasimhan, Springer-Verlag, Berlin 1990, 472-476. Zbl 0703.01020 MR 1084595

[Sch] F. Schottky, Ueber die conforme Abbildung mehrfach zusammenhängender ebener Flächen. J. Reine Angew. Math. 83 (1877), 300-351. JFM 09.0287.01

[Tie] H. Tietz, Eine Normalform berandeter Riemannscher Flächen. Math. Ann. 129 (1955), 44-49. Zbl 0064.07702 MR 0069276

[Vi] J. W. Vick, Homology Theory : An Introduction to Algebraic Topology. 2nd ed., Grad. Texts in Math. 145, Springer-Verlag, Berlin 1994. Zbl 0789.55004 MR 1254439

Received May 10, 2005

Alexandre Gabard, Université de Genève, Section de Mathématiques, 2-4, rue du Lièvre, CP 240, 1211 Genève 24 Suisse

E-mail: alexandregabard@hotmail.com 